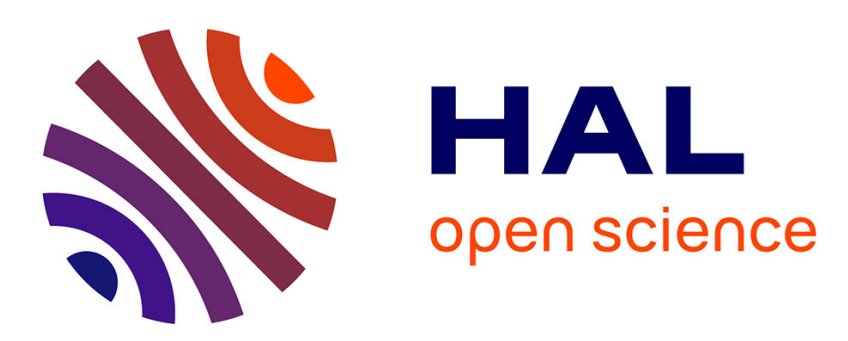

\title{
Non-linear Effect Mitigation for FMCW Radar System
}

Nizar Bouhlel, Stéphane Meric, Claude Moullec, Christian Brousseau

\section{To cite this version:}

Nizar Bouhlel, Stéphane Meric, Claude Moullec, Christian Brousseau. Non-linear Effect Mitigation for FMCW Radar System. European Radar Conference (EuRAD 2018), Sep 2018, Madrid, Spain. 10.23919/eurad.2018.8546550 . hal-01894265

\section{HAL Id: hal-01894265 https://hal.science/hal-01894265}

Submitted on 12 Oct 2018

HAL is a multi-disciplinary open access archive for the deposit and dissemination of scientific research documents, whether they are published or not. The documents may come from teaching and research institutions in France or abroad, or from public or private research centers.
L'archive ouverte pluridisciplinaire HAL, est destinée au dépôt et à la diffusion de documents scientifiques de niveau recherche, publiés ou non, émanant des établissements d'enseignement et de recherche français ou étrangers, des laboratoires publics ou privés. 


\title{
Non-linear Effect Mitigation for FMCW Radar System
}

\author{
Nizar Bouhlel $^{1}$, Stéphane Méric ${ }^{1}$, Claude Moullec ${ }^{2}$, Christian Brousseau $^{1}$ \\ ${ }^{1}$ Institut d'électronique et de télécommunications de Rennes, IETR, France \\ ${ }^{2}$ ADVANTEN, 30 route de Fougères, Cesson-Sévigné, France
}

\begin{abstract}
In this paper, we deal with a frequency modulated continuous wave (FMCW) radar used for localizing and tracking targets by frequency evaluation of the received radar beat signal. The radar system achieved with a primary radar (reader) and a secondary radar (transponder) is addressed as super high frequency (SHF) radio frequency identification (RFID). Consequently, considering the transponder as an active target, we achieve an identification application thanks to the shift frequency induced by the transponder. Moreover, the impact of the non-linearity behavior of this transponder on the localization performance, is investigated and a solution is proposed for cancelling non-linear effects.
\end{abstract}

Keywords - Frequency-modulated continuous wave (FMCW), RFID transponder, signal processing, distance and shift frequency measurements, range-frequency map

\section{INTRODUCTION}

The frequency-modulated continuous wave (FMCW) radars are systems transmitting a continuous and frequency modulated waveform [1]. These radars transmit and receive simultaneously and without interruption unlike modulated pulsed radars. The FMCW radars are widely used for industrial and academic with many useful applications [2] such as as snow thickness measurement [3], imaging application like SAR imagery [2], terrain displacement monitoring [4], as well as automotive applications in collision avoidance [5]. An FMCW radar is used to locate targets [6], [7], [2] since it can detect the ranges and velocities of objects. Another way to remotely detect object is the use of the radio frequency identification (RFID) systems. The RFID allows identification and tracking of persons or objects. With the technological advances of RFID it is possible to operate with UHF and SHF range allowing a larger operating distance [8], [9]. Two types of RFID systems are still used nowadays: passive and active. The passive UHF transponders can operate up to a distance of 10 meters. The active transponders with their own battery allow higher distances for detection and characterization applications. In the case of active configuration, the connected object is considered as a cooperative target. Thus, we can combine the RFID concept that considers the target as an active device to radar systems which can evaluate the received radar beat signal for localizing and tracking targets.

In this paper, we deal with the system GESTAR (GEnerator System for TArget Radar identification). This system is a radar system for detecting and localizing cooperative targets. The system is composed of a small reader (the primary radar) and a shift frequency identification transponder (the secondary radar) responding to the primary radar only when this latter produces an interrogation signal. Indeed, the primary radar transmits a FMCW that is received by the transponder. The transponder re-transmits this signal by introducing its own shift frequency. The power transmitted by the transponder is captured by the primary radar. After signal processing, the target (transponder) is then precisely located by using a constant false alarm rate (CFAR) detection. Finally, the reader displays on a small screen the position (radial distance), the angular position and the shift frequency of the targets. The particularity and the innovation of GESTAR is the absence of blind distance as well as the great autonomy of the transponder. The performances of this system are characterized by several parameters namely: the range resolution, the shift frequency resolution and the maximum range detection capacity.

In this study, the used transponder operates a non-linear modulation. In this case, the range-shift frequency map is actually affected by the presence of several harmonics that are added to the spectrum and caused by the aliasing. Therefore, the identification of the target becomes difficult. We propose in this paper to study the effect of non-linearity behavior of the transponder in the detection and localization. Moreover, we present a method to remove the undesirable harmonics validated by simulations and measurements. The paper is organized as follows. Section II introduces the principles of RFID localization based on FMCW signal, the non linearity behaviors of the transponder and the consequences on the detection and localization. Section III presents some simulation of removing undesirable harmonics. Some real measurements are shown in section IV. Finally, section V concludes this paper.

\section{RFID LOCALIZATION BASED ON FMCW SIGNAL}

Fig. 1 shows the principle of the RFID localization system using FMCW radar system. The primary radar system transmits a signal $s_{e}(t)$ assumed to be a linear frequency modulated signal in time interval $[0, T]$. The analytic expression of $s_{e}(t)$ is given as follows

$$
s_{e}(t)=A_{e} \exp j 2 \pi\left(f_{0} t+\frac{B}{2 T} t^{2}\right)
$$

where $A_{e}, f_{0}, B$, and $T$ denote respectively the amplitude, the RF carrier frequency, the sweep bandwidth, and the sweep duration (the modulation duration). The RFID transponder, considered as a secondary radar and located at distance 


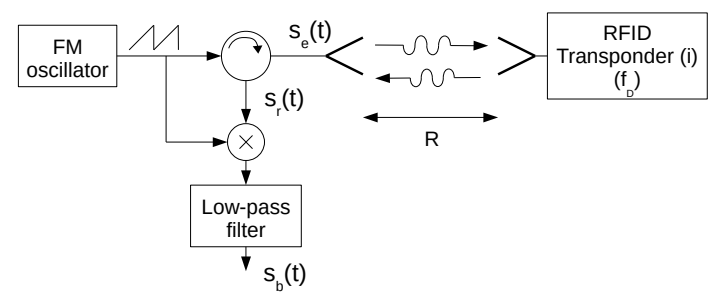

Fig. 1. Setup of FMCW radar based RFID localization system

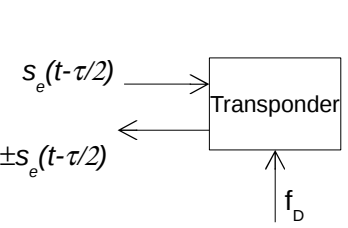

(a)

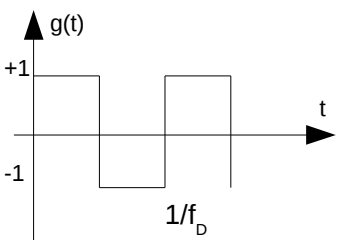

(b)
Fig. 2. Modeling the behavior of the non-linear transponder.

$R$ from the primary radar, receives the transmitted radar signal with a delay of $\tau / 2=R / c$ where $c$ is the velocity of the wave. Consequently, the transponder modulates this received signal by its proper signal $g(t)$ as it is shown in Fig. 2.a. The waveform $g(t)$ is considered as the transponder characteristic. For our study, we propose to modelize the non-linear transponder effect with an alternative periodic signal $g(t)$ of $1 / f_{D}$ period. Fig. 2.b shows $g(t)$ as function of time. Since $g(t)$ is periodic, we can derive it into Fourier series

$$
g(t)=\sum_{n=-\infty}^{+\infty} C_{n} \exp \left(j 2 \pi n f_{D} t\right)
$$

where the complex coefficient $C_{n}$ is given by

$$
C_{n}=f_{D} \int_{0}^{1 / f_{D}} g(t) \exp \left(-j 2 \pi n f_{D} t\right) d t=\frac{2}{j \pi n}, \quad(n \text { odd })
$$

The received signal by the reader (primary radar) $s_{r}(t)=$ $g(t) . s_{e}(t-\tau)$ is an attenuated and delayed version of the transmitted signal with a delay of $\tau$. The principle of an homodyne demodulation of the received signal consists in mixing the signal $s_{e}(t)$ with the received signal $s_{r}(t)$. The expression of the beat signal at the mixer output is $s_{b}(t)=$ $s_{e}(t) s_{r}^{*}(t)$ where $\left.{ }^{*}\right)$ denotes the conjugate operator. The delay between the transmitted signal and the received signal results in a constant beat frequency called $f_{b}$ which makes possible to measure the distance between the target (transponder) and the radar. The beat signal is given as follows

$$
s_{b}(t)=g(t) \cdot \exp \left(j 2 \pi\left(f_{b} t+\phi_{0}\right)\right)
$$

where the beat frequency $f_{b}=B \tau / T=2 B R /(c T)$ and $\phi_{0}=$ $f_{0} \tau-B /(2 T) \tau^{2}$ is a constant phase. The knowledge of the value of $f_{b}$ allows the determination of $R$.

In case of a frequency shift induced in the received signal, a FMCW radar can also measure this frequency shift $f_{D}$ by integrating data from several cycles of wave replication as

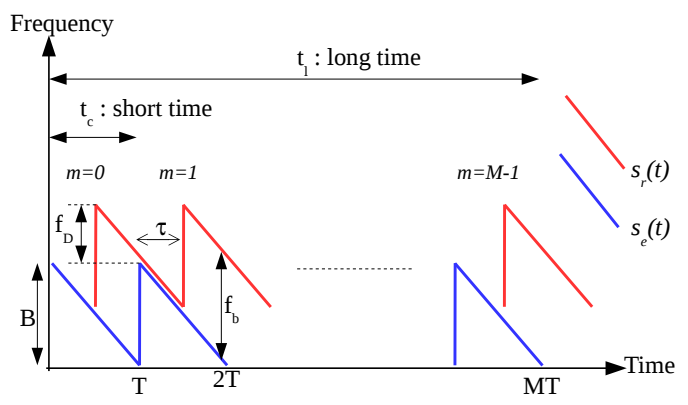

Fig. 3. Beat signal over several cycles

shown in Fig. 3. Then, we define the short time $t_{c}$ as the time description during the sweep duration $T$. The long time axis $t_{l}$ is defined as a time data set that collects one sample for each $T$ duration: $t_{l}=m T$ with $m=0,1, \ldots, M-1(M$ is the total number of modulation duration). Consequently, the beat signal $s_{b}(t)$ could be described both with respect to the time axis $t_{c}$ and $t_{l}$

$$
s_{b}\left(t_{c}, t_{l}\right)=g\left(t_{c}+t_{l}\right) \cdot \exp \left(j 2 \pi\left(f_{b} t_{c}+\phi_{0}\right)\right)
$$

To establish the range-shift frequency map, the beat signal analysis is carried out in the Fourier domain. We recall that the short time $t_{c}$ is sampled according to the sampling frequency $F_{e}$, and the long time $t_{l}$ according to the period repetition frequency $F_{s}=1 / T$. The two-dimensional Fourier transform $S_{b}\left(f, f^{\prime}\right)$ of the sampled beat signal (according to $F_{e}$ and $F_{s}$ respectively) and that of the two-dimensional Fourier $S_{b}^{c}\left(f, f^{\prime}\right)$, where the exposant $c$ means the continuous time, are connect by the Poisson formula given by

$$
S_{b}\left(f, f^{\prime}\right)=F_{e} F_{s} \sum_{n=-\infty}^{\infty} \sum_{k=-\infty}^{\infty} S_{b}^{c}\left(f-n F_{e}, f^{\prime}-k F_{s}\right)
$$

The first Fourier transform is calculated for $\tau_{\max }<t_{c}<T$ where $\tau_{\max }$ corresponds to the delay for a maximum distance. The second transform is calculated for $0<t_{l}<(M-1) T$. Since an anti-aliasing filter is applied in range frequency, the first term $n F_{e}$ is omitted and the expression can be reduced as follows:

$$
\begin{aligned}
& S_{b}\left(f, f^{\prime}\right)=F_{e} F_{s} e^{j 2 \pi \phi_{0}} A B \sum_{k=-\infty}^{\infty} \sum_{n=-\infty}^{\infty} C_{n} \\
& \times e^{-j \pi\left(f^{\prime}-k F_{s}-n f_{D}\right) A_{1}} \operatorname{sinc}\left(\pi\left(f^{\prime}-k F_{s}-n f_{D}\right) A\right) \\
& \times e^{-j \pi\left(f-f_{b}-n f_{D}\right)\left(T+\tau_{\max }\right)} \operatorname{sinc}\left(\pi\left(f-f_{b}-n f_{D}\right) B\right)
\end{aligned}
$$

where $A=(M-1) T$ and $B=T-\tau_{\max }$. Fig. 4 shows the range-shift frequency map as the spectrum magnitude of the function (7) where the variable $f$ represents the beat frequency i.e. the target position, and $f^{\prime}$ the shift frequency. The spectrum magnitude corresponds to the configuration where the transponder is located at the distance $50 \mathrm{~m}$ meaning that $f_{b}=250 \mathrm{kHz}$. Moreover, the shift frequency is $f_{D}=19.4 \mathrm{kHz}$. In a global way, we can consider that the received spectrum could exhibit several harmonics that would be due on the one hand to the aliasing effect 


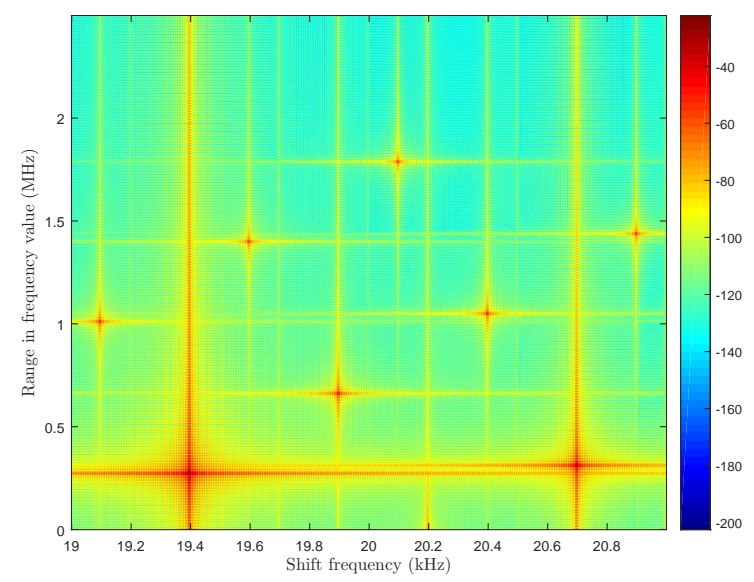

Fig. 4. Two-dimensional discrete-time Fourier where sampling frequencies are $F_{e}=20 \mathrm{MHz}$ and $F_{s}=12.5 \mathrm{kHz}$. The shift frequency of the non-linear transponder is $f_{D}=19.4 \mathrm{kHz}$. The intervals are [0, 2.5 MHz] for the distance and $[19 \mathrm{kHz}, 21 \mathrm{kHz}]$ for the shift frequency.

according to shift frequencies since we use the interval between $12.5 \mathrm{kHz}$ and $25 \mathrm{kHz}$ and on the other hand to the non-linear transponder effects. In our application, the cut-off frequency of $3 \mathrm{MHz}$ is used for the anti-aliasing filter, that does not allow aliasing in the $[0,3 \mathrm{MHz}]$ band and does not induce effect to the range response. Nevertheless, considering Fig. 4, many harmonics are detected along the shift frequency that corresponds to the a non-linear transponder behavior. It is obvious that these harmonics are not connected to any transponder. These harmonics correspond to the maximums reached by the functions $\operatorname{sinc}\left\{\pi\left(f-f_{b}-n f_{D}\right)\left(T-\tau_{\max }\right)\right\}$ and $\operatorname{sinc}\left\{\pi\left(f^{\prime}-k F_{s}-n f_{D}\right)(M-1) T\right\}$ for frequencies $f=f_{b}+n f_{D}$ and $f^{\prime}=k F_{s}+n f_{D}$. These harmonics increase the false alarm rate that is dramatically useless for a detection system as GESTAR. We can precisely analyse the range-shift frequency map which is exhibited in Fig. 4. In the one hand, the real position of the transponder is that of the position of the fundamental characterized by $(k, n)=(0,1)$ with range frequency $f=f_{b}+f_{D}=269.4 \mathrm{kHz}$, meaning that the detected range is $54.01 \mathrm{~m}$, and the shift frequency $f^{\prime}=f_{D}=19.4 \mathrm{kHz}$. The position along the shift frequency axis is correct but the range value is biased by an additional frequency $f_{D}$. This is taken into account in the radar software by subtracting $f_{D}$ from the range frequency. In the other hand, the harmonics visible in the magnitude of the spectrum and caused by the non-linear transponder behavior, strongly affect the detection and the location of the real targets because they are considered themselves as targets and then gives erroneous interpretations. The solution to this problem is to suppress these harmonics by calculating their positions. In the following subsection, we present a solution to remove the harmonics.

\section{SIMULATION OF REMOVING UNDESIRABLE HARMONICS}

To remove the aliasing effects that induce false alarms, we propose to modify the modulation time $T$. Indeed, from the expression of the bidimensional Fourier transform $S_{b}\left(f, f^{\prime}\right)$ of the beat signal given by (7), the position of the harmonics depends on the modulation time $T=1 / F_{s}$. The idea is to
Table 1. Comparison between the ground truth values and the assessed parameters (angles and ranges). Measurements of shift frequencies and the SNR $(\mathrm{dB})$ are added.

\begin{tabular}{cc|cccc}
\hline \hline \multicolumn{2}{c|}{ Ground thruth } & \multicolumn{4}{c}{ Estimation from Measurements } \\
\hline Angle & Range & Angle & Range & Shift & SNR \\
$\theta(\mathrm{rad})$ & $R(\mathrm{~m})$ & $\hat{\theta}(\mathrm{rad})$ & $\hat{R}(\mathrm{~m})$ & frequency $(\mathrm{kHz})$ & $(\mathrm{dB})$ \\
\hline \hline \multirow{2}{*}{0} & 139.6 & 0.0001 & 135 & 19.4 & 39.17 \\
& 115.1 & -0.0002 & 117.5 & 19.4 & 37.48 \\
& 83.4 & 0.0001 & 85 & 19.4 & 42.23 \\
& 52.1 & 0.0000 & 52.5 & 19.4 & 48.53 \\
\hline \multirow{2}{*}{0.1053} & 115.74 & 0.0958 & 117.5 & 19.4 & 35.95 \\
& 52.9 & 0.1564 & 52.5 & 19.4 & 46.42 \\
\hline 0.1946 & 117.31 & 0.0075 & 120 & 19.4 & 28.81 \\
\hline 0.3015 & 120.54 & 0.0024 & 125 & 19.4 & 16.74 \\
\hline \hline
\end{tabular}

use several values of modulation time $T$ and then to multiply two or more range-shift frequency maps corresponding to these different values of $T$. The fundamental frequency corresponding to the true target is retained by the product and the harmonic frequencies are attenuated. It should be noted that the range resolution remains unchanged since it is given by $\Delta R=\frac{c}{2 B}$ where $B$ is the transmitted signal bandwidth. However, the shift frequency resolution changes since it is inversely proportional to the observation time of the processed signal $\Delta f_{D}=\frac{1}{M T}$ where $M$ is the repetition number of the modulation time. To avoid a big change in the shift frequency resolution, the used modulation times are very close to each other. In this way, one does not affect too much the shift frequency resolution. The proposed method is applied to various conditions and some simulations are presented here. The Fig. 5 shows a configuration of transponder location where the position of the transponder is at $R=85 \mathrm{~m}$ from the reader. The shift frequency is $f_{D}=19.4 \mathrm{kHz}$. Each simulation corresponds to a different time modulation $T=\{80 \mu s, 80.1 \mu s, 80.2 \mu s\}$. As shown in Fig. 5.a, .b and .c, the harmonics are presents in all the examples and may be confused with targets. As noted before, the position of these harmonics correspond to the maximums reached by $S_{b}\left(f, f^{\prime}\right)$ for $f=f_{b}+n f_{D}$ and $f^{\prime}=k F_{s}+n f_{D}$. It is clear that the multiplication of two or three images strongly attenuates the harmonics and retains only the fundamental that corresponds to the real target as it is shown in Fig. 5.d.

\section{OUTDOOR MEASUREMENTS}

Outdoor measurement series are carried out in an open place. The transponder is placed directly in front of the radar at different ranges and with different offset angles ranging from 0 radian to 0.3 radian. The shift frequency of the transponder is set at $f_{D}=19.4 \mathrm{kHz}$. Table 1 describes different configurations for the transponder positions (range $R$ and angle $\theta$ ) and gives the estimated distances $\hat{R}$, angles $\hat{\theta}$ and shift frequency. The signal-to-noise ratio (SNR) is given to see its evolution as a function of distance and orientation. According to these measurements, we can note 


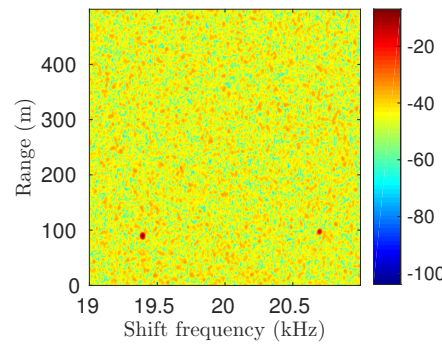

(a) $T=80 \mu \mathrm{s}$

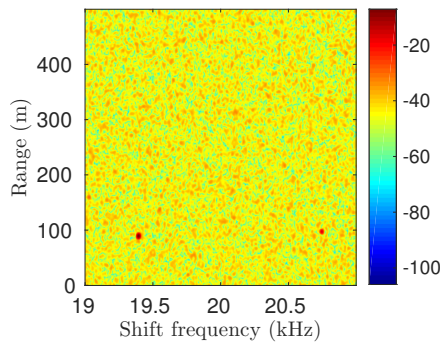

(b) $T=80.1 \mu \mathrm{s}$

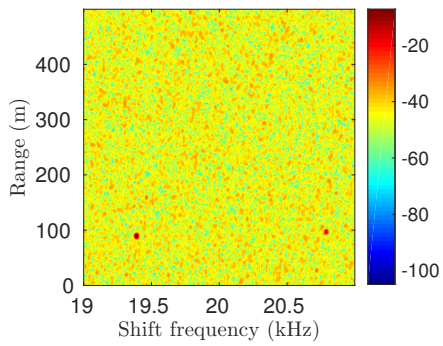

(c) $T=80.2 \mu \mathrm{s}$

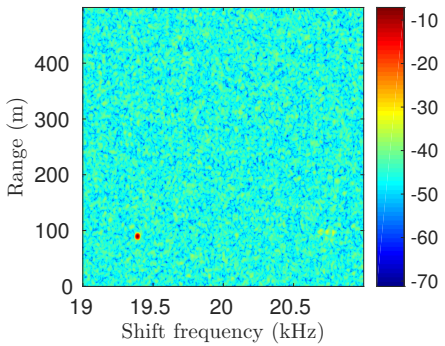

(d) product

Fig. 5. Simulations of range-shift frequency map with different modulation times $T=[80,80.1,80.2] \mu$ s given respectively in (a), (b) and (c). The distance between transponder and reader is $R=85 \mathrm{~m}$.

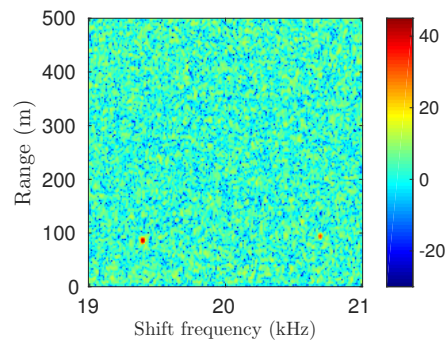

(a) $T=80 \mu \mathrm{s}$

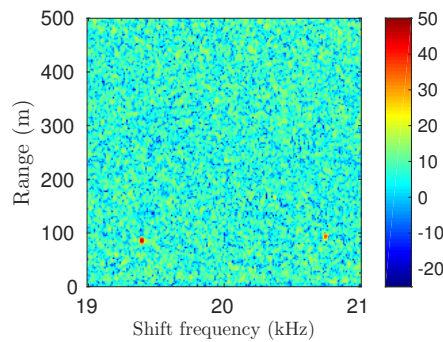

(b) $T=80.1 \mu \mathrm{s}$

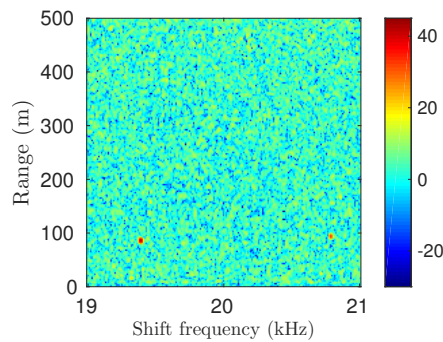

(c) $T=80.2 \mu \mathrm{s}$

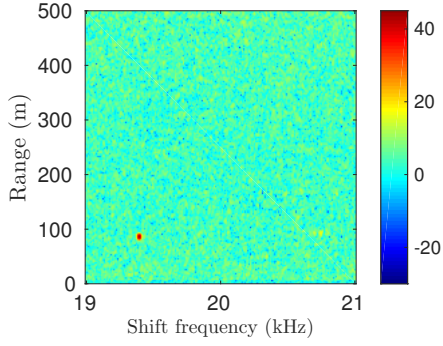

(d) product

Fig. 6. Range-shift frequency map provided from real measurements for a distance $R=85 \mathrm{~m}$ and with different modulation times $T=\{80,80.1,80.2\} \mu$ s. The figure (d) is the product of range-shift frequency maps given by (a), (b) and (c).

that the estimated shift frequency is the same as the real shift frequency which characterizes the transponder. Moreover, the estimated ranges are close to the ground truth distances between the transponder and the reader. The error rate is low for short distances and increases as distance increases. The angle assess remains reliable both for near-zero orientation angles and for small distances up to $52.5 \mathrm{~m}$. In Fig. 6, we show range-shift frequency maps provided by the radar system for three modulation times for a transponder position $R=85 \mathrm{~m}$. It is clear that we have the same behavior with the simulations exhibited in Fig. 5 i.e. the same location of the harmonics. The product of these maps realized for different modulation times makes possible to attenuate the harmonics and to keep the fundamental that corresponds to the real target to be detected. Fig. 6.d corresponds to the product of the range-shift frequency map of Fig. 6.a, .b and .c.

\section{CONCLUSION}

In this paper, a FMCW radar system for RFID identification is presented. The range-shift frequency map is carried out from the spectral analysis of the beat signal. We define a non-linear transponder where the shift frequency is non-linearly added to the transmitted frequency. Also, the assessment of the performance of the systems are presented. The effects from the non-linear behavior of the transponder on the beat frequency spectrum are studied. Furthermore, a signal processing procedure is proposed to mitigate and remove false alarms. The validation of the solutions are presented and validated based on simulated and real FMCW radar data. In perspective, we project the use of other transmitted waveforms as step frequency continuous wave (SFCW) in order to enhance the signal-to-noise ratio.

\section{ACKNOWLEDGMENT}

The authors would like to thank the DGA (Direction Générale de l'Armement) for funding this study.

\section{REFERENCES}

[1] A. Stove, "Linear fmcw radar techniques," IEE Proceedings F (Radar and Signal Processing), vol. 139, pp. 343-350(7), October 1992.

[2] J. W. Ting, D. Oloumi, and K. Rambabu, "Fmcw sar system for near-distance imaging applications-practical considerations and calibrations," IEEE Trans. Microw. Theory Techn., vol. PP, no. 99, pp. $1-12,2017$.

[3] N. Galin, A. Worby, T. Markus, C. Leuschen, and P. Gogineni, "Validation of airborne fmcw radar measurements of snow thickness over sea ice in antarctica," IEEE Trans. Geosci. Remote Sens., vol. 50, no. 1, pp. 3-12, Jan 2012.

[4] R. Iglesias, A. Aguasca, X. Fabregas, J. J. Mallorqui, D. Monells, C. Lpez-Martnez, and L. Pipia, "Ground-based polarimetric sar interferometry for the monitoring of terrain displacement phenomena x2013;part ii: Applications," IEEE J. Sel. Topics Appl. Earth Observ. Remote Sens., vol. 8, no. 3, pp. 994-1007, March 2015.

[5] B. Boukari, E. Moldovan, S. Affes, K. Wu, R. G. Bosisio, and S. O. Tatu, "Six-port fmcw collision avoidance radar sensor configurations," in 2008 Canadian Conference on Electrical and Computer Engineering, May 2008, pp. 305-308.

[6] Y. Al-Alem, L. Albasha, and H. Mir, "High-resolution on-chip $s$-band radar system using stretch processing," IEEE Sensors Journal, vol. 16, no. 12, pp. 4749-4759, June 2016.

[7] Y. Wang and Y. Zheng, "An fmcw radar transceiver chip for object positioning and human limb motion detection," IEEE Sensors Journal, vol. 17, no. 2, pp. 236-237, Jan 2017.

[8] L. Ghattas, S. Mric, and C. Brousseau, "Performance assessment of fmcw radar processing for transponder identification," in 2016 European Radar Conference (EuRAD), Oct 2016, pp. 258-261.

[9] D. Hazry, R. M. S. Muhammad, and S. Zairi, "Study of integration 2.4ghz and 5.8ghz in rfid tag," Proceedings of the International Conference on Man-Machine Systems (ICoMMS), 102009. 